\title{
Understanding Behaviour of T shaped Building under Seismic loading
}

\author{
N.W.Mankar ${ }^{1}$, Prof. Dr.A.M.Pande ${ }^{2}$ \\ PhD scholar, Department of Civil Engineering, Yeshwantrao Chavan College of Engineering, \\ Nagpur University, Nagpur, Maharashtra India \\ Professor, Department of Civil Engineering. \& Director $R \& D$, Yeshwantrao Chavan College of Engineering, \\ Nagpur, Maharashtra, India.
}

\begin{abstract}
During an earthquake the behavior of the building is on its overall shapes, size \& geometry. Now a day's many buildings are asymmetric in plan and elevation. Due to irregular distribution of mass, stiffness and strengths may cause serious damage in structural system. There are various types of irregularities in the buildings depending upon their location and scope. Mainly two types of irregularities as per IS -1893(Part-I)2002. a) plan or horizontal irregularities b) vertical irregularities. Irregularity in structure makes analysis of the seismic behavior very complicated. Present work is on a T shape building, the rotational flexibility on the T shape building and a part of $T$ shape building reduced from $100 \%$ to $0 \%$. The soft computing tool and commercial software STAAD-Pro is used for modeling and analysis. Building plans of odd shapes shows an erratic development of forces. By introducing the rotational flexibility forces can be reduced to some level, however precaution is necessary while introducing rotational flexibility.
\end{abstract}

Keywords: Asymmetric Structure, Irregular profile, Torsion, RSM, IS -1893(Part-I)-2002.

\section{Introduction}

Earthquake produces waves which vibrate the base of structure in various manners and directions, because of which lateral force is developed on structure which shears the column or sometimes even it buckles the whole column resulting in failure of structure, stiffness is the ability of a component or an assembly of components to resist deformations when subjected to actions. It is expressed as the ratio between action \& deformation at a given level of either of the two quantities and the corresponding value of the other. IS 1893 (part-1) :2002 [1] has recommended building configuration as regular or irregular in terms of the size and shape of the building, arrangement of structural elements and mass during earthquake.

\section{Review of literature}

Mohit Sharma, et.al, in this paper RCC frames structure is analyzed both statically and dynamically. It is seen that to develop a simple analytical procedure based on rigorous computations and experiments on the seismic response of irregular structures is necessary. [1]

Rakesh Sakale, et.al showed that in building having horizontal irregularities in all zones parameters like displacement \& storey drift follows the permissible limits as per coda provisions. While story drift, drift values are maximum near the second to tenth storey. While displacement shear wall is required to control the permissible limit. [2]

Rucha S. Banginwar, et.al has overviewed Structural configuration play an important role in seismic performance depends on the building configuration by considering scale, height, horizontal size, proportion \& symmetry of building. It has been concluded that plan configuration has significant impact on the seismic response of structure in terms of displacement, storey drift, story shear \& building with severe irregularity shows maximum displacement and story drift. [3]

Divyashree M, et.al the building with re-entered corners causes abrupt changes in the strength or stiffness of the structure causes the rotation in the building. Thus convert the shape in to smaller regular shapes is one of the solutions. One of the methods to strengthen the re-entrant corner using elements such as shear wall, bracing, or mild steel splays. [4]

\section{Work Done}

The structural analysis of a five story reinforced concrete building is done in the present work. Two typical buildings building as a complete $\mathrm{T}$ shape and building as a part of $\mathrm{T}$ shape for comparison of their seismic performance. On complete building forces acting on $\mathrm{T}$ shape building and building a part of $\mathrm{T}$ shape as regular building with forces acting on one side of building i.e. edge CF. For analysis considering $100 \%, 75 \%$, $50 \% \& 25 \%$ forces of the original forces acting on this part when it is complete T shape building and the result of this variation on the building on front side, middle side $\&$ back side of the building. 
The main objective of the present work is to study the variation in the behavior of $\mathrm{T}$ shaped building and as a part of $\mathrm{T}$ shape building and compare the behavior of columns after applying different forces percentagewise on one side and effect on front, middle \& back row of column of as-symmetrical (T shape \& part of T shape ). Exact behavior of flange and web parts of $\mathrm{T}$ shaped building is not yet investigated.

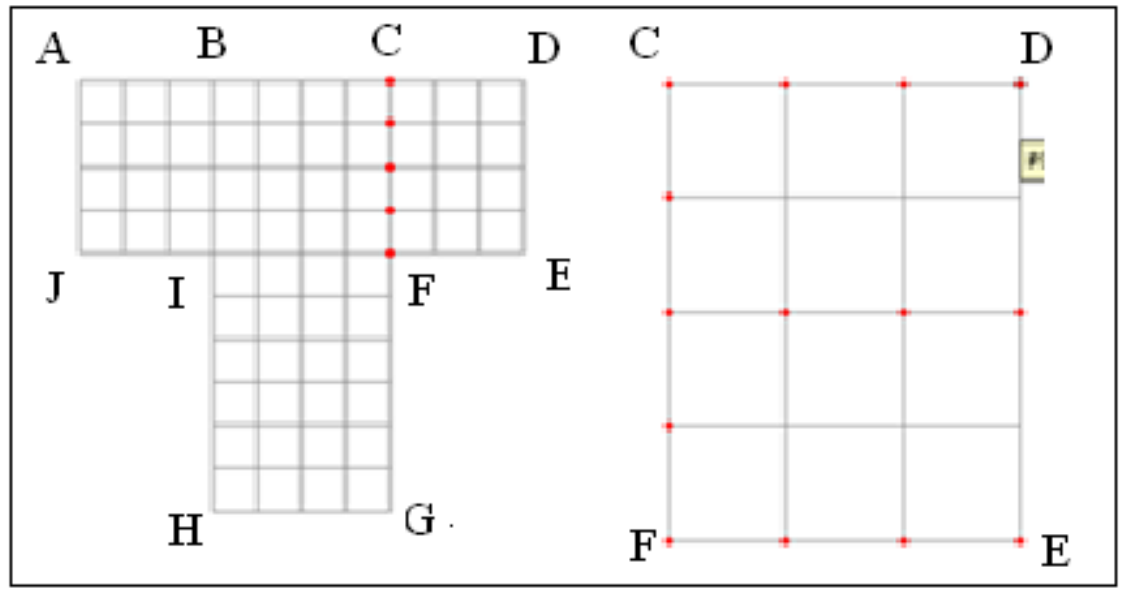

FIG. 1 T SHAPE FRAMES COMPLETE \& A PART

Two frames are selected as shown in fig, 1. \& 2, for the analysis of five storied building following dimensions are considered which are elaborated below. Building structural data in order to understand behavior of structure of non realistic building configuration is chosen.

Table 2: Structural details

Building Details Shape of building: T shape

No of Floors : : five storey's

Purpose of Building: Residential

Ground Floor : $\quad 2.00 \mathrm{mt}$.

Storey Height $\quad$ : $\quad 3.00 \mathrm{mt}$.

Length of Building: $\quad 40.00 \mathrm{mt}$.

Width of Building: $\quad 40.00 \mathrm{mt}$.

Slab Thickness : $125 \mathrm{~mm}$

Wall thickness : $\quad 230 \mathrm{~mm}$

Beam size : $\quad 230 \times 300 \mathrm{~mm}$

Column size : $\quad 230 \times 400 \mathrm{~mm}$

Concrete Grade : $\quad$ M-20

Steel Grade : : Fe-415

Density of Concrete: $\quad 25.00 \mathrm{kn} / \mathrm{m}^{3}$

Density of brick : $\quad 20.00 \mathrm{kn} / \mathrm{m}^{3}$

Live load : $\quad 2.00 \mathrm{kn} / \mathrm{m}^{2}$

Roof live load : $\quad 1.50 \mathrm{kn} / \mathrm{m}^{2}$

Floor Finish : $\quad 1.00 \mathrm{kn} / \mathrm{m}^{2}$

\section{Earthquake Data}

Seismic Zone : II

Response Reduction Factor: $\quad 3.0$

Importance factor $\quad: \quad 1.0$

Soil site factor-Medium : $\quad 2.0$

Type of Structure- Frame: $\quad 1.0$

Damping Ratio $\quad$ : $\quad 5.0 \%$

Period of Structure in X Dir.: $\quad 3.0$

Period of Structure in Z Dir: $\quad 0.5$

For the analysis purpose, the structure is assumed to be in zone -II (zone factor 0.1) on the site with medium soil. These structures are taken as general building and hence importance factor is taken as 1 , and the frame is proposed to have ordinary RC moment resisting frames and hence the reduction factor is taken as 3 . In this case it is proposed to take as building structure with coefficient of exposure 0.8 , response of the building 
frame structures is studied mainly for the dominated load combination in Z- direction for the elected columns at different levels including displacement.

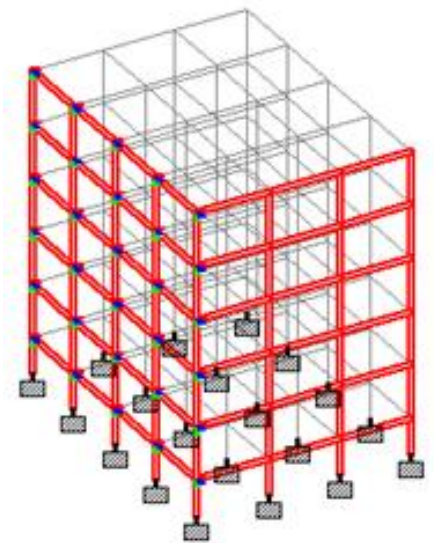

Front

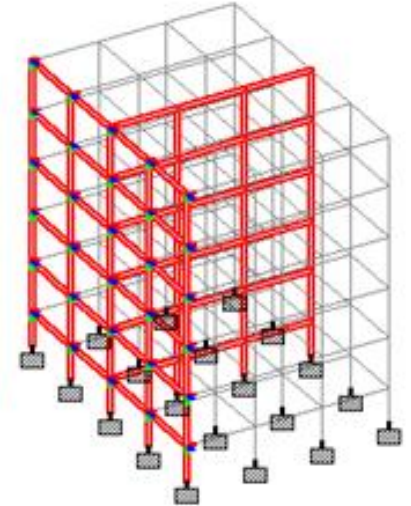

Middle

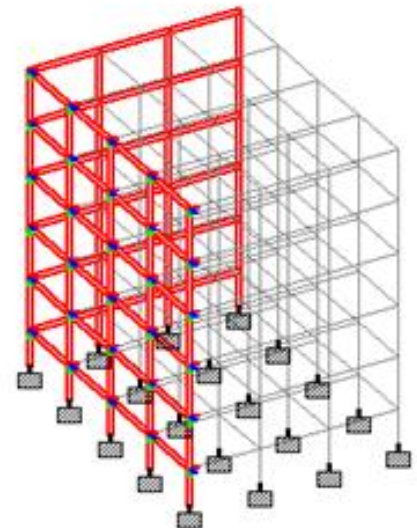

Back

\section{FIG.2 FRONT, MIDDLE \& BACK ROW OF PART OF T SHAPE BUILDING}

\section{Result / Discussion}

Observation based on inducing rotational freedom in flange part of $\mathrm{T}$ section buildings, when the earthquake forces are applied in $\mathrm{Z}$ direction

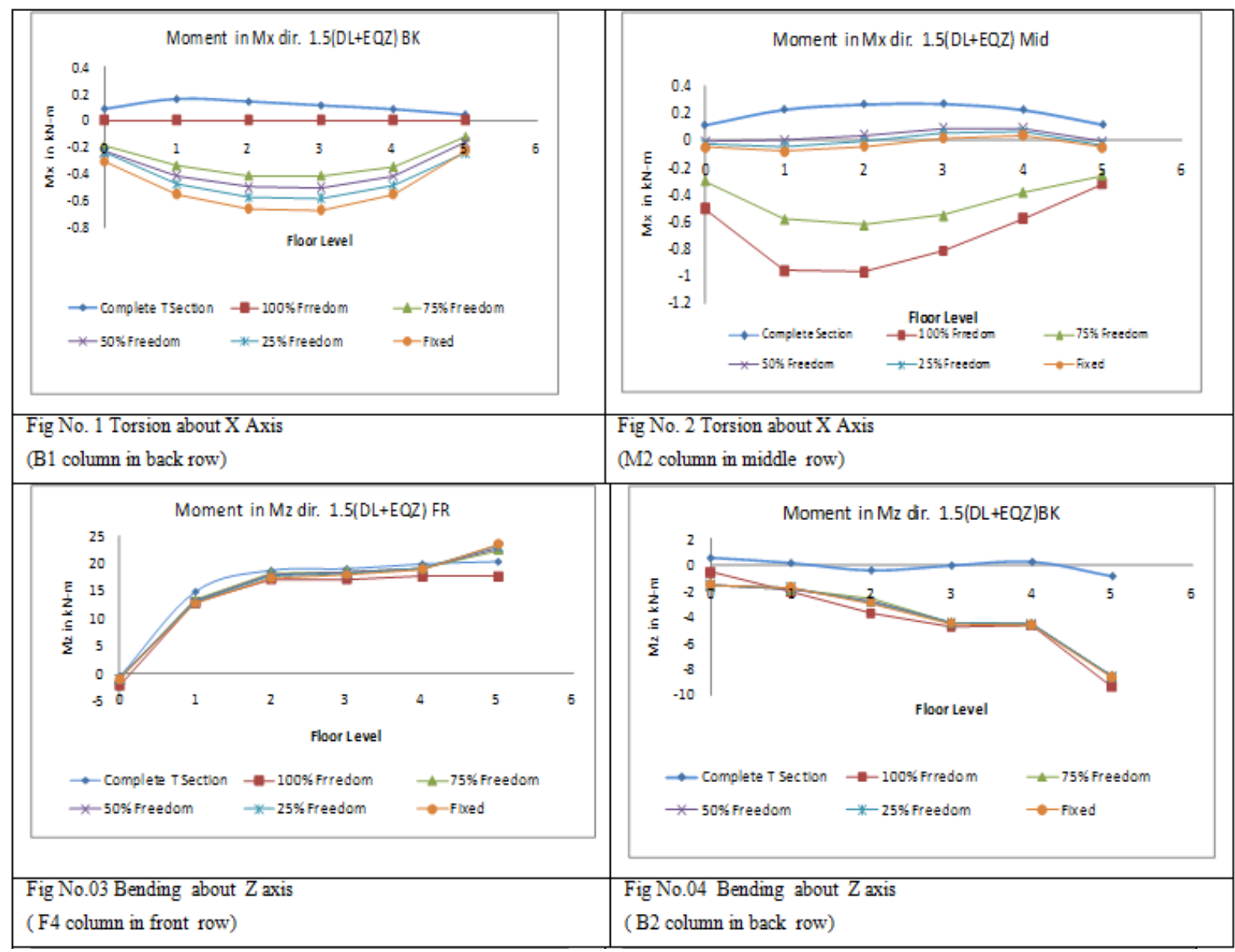




\begin{tabular}{|l|l|l|l|}
\hline \\
Fig No. 05 Shear Forces about Y axis \\
(F3 column in front row)
\end{tabular}

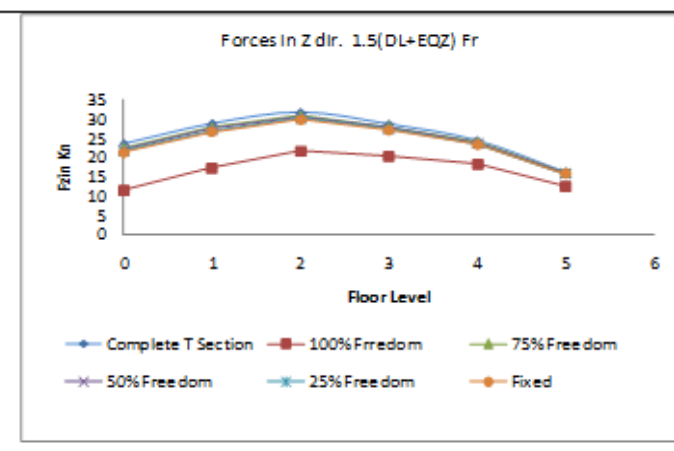

Fig No.7 Shear forces about $\mathrm{Z}$ axis

( F4 column in front row)

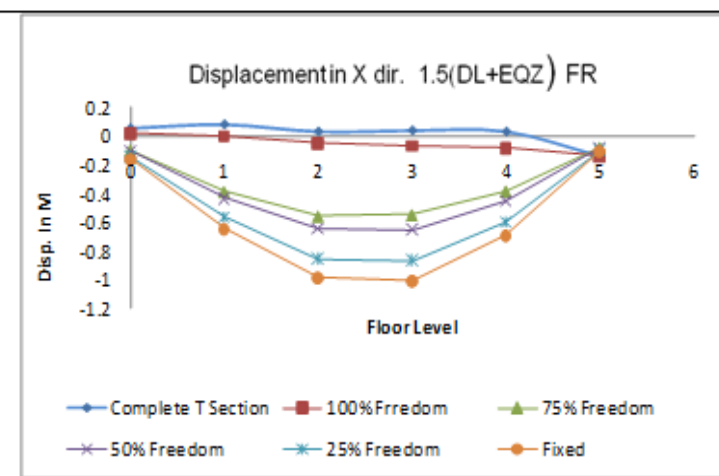

Fig No.8 Shear forces about $\mathrm{Z}$ axis

( M3 column in middle row)
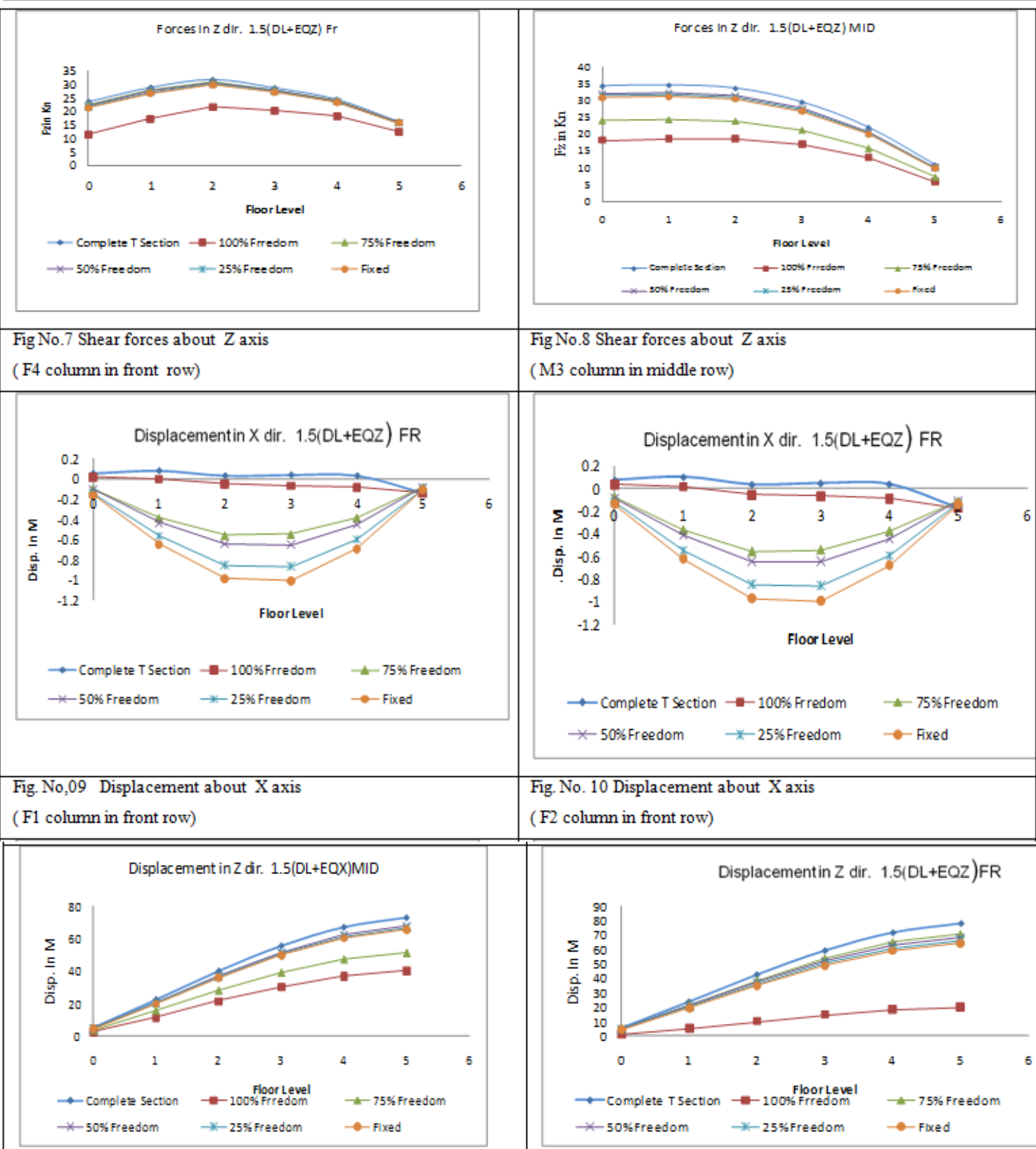

( $F 2$ column in front row)

Fig. No, 11 Displacement about $\mathrm{Z}$ axis

( $F 1$ column in front row)

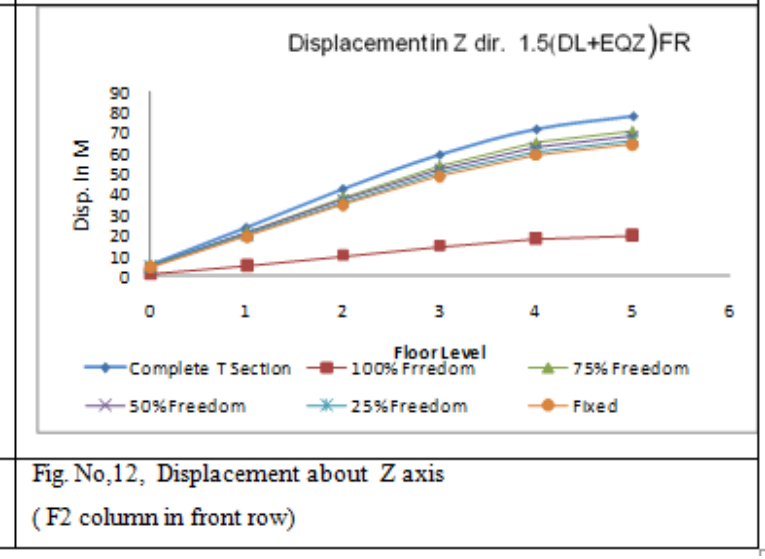


1. Back row of columns, with the increasing rotational freedom the torsion about X-axis is relatively higher for Columns very close to the section connecting flange and web. However this goes on reducing towards end of flange in X-direction.100\% freedom may result in much higher torsion as indicated by fig $1 \&$ fig 2 .

2. In the middle row of column similar trends are seen however at $75 \% \& 100 \%$ freedom levels torsion is much higher as indicated by fig 2 .

3. In the front row of column the behavior is similar to back row however in the outermost column of this row the behavior is beat erratic resulting in higher amount of torsion in the top floor as indicated by fig. no. $3 \& 4$

4. Bending about $\mathrm{Z}$ direction is significant higher for column close to connection between web \& flange it goes on reducing toward end of flange for all freedoms, No significant changes observed in axial forces observed.

5. Significant increase in shear in transverse direction is observed for column very close to the connection, it goes on reducing towards the end of flange as indicated in Fig. $5 \& 6$

6. The front \& middle lines of column exhibits the significant difference in the development of shear in $Z$ direction these freedom in rotation is very significant in reducing shear force in middle row. Similar trends in the back row.

7. The rotational freedom increases the displacement at floors between the top and ground floor in all types of column Fig. 9\& 10.

8. The freedom in the rotation reduces displacement in the middle level of column, while in back and front row there is no significant reduction in displacement. Fig. $11 \& 12$.

\section{Concluding Remark}

1) Building plans of odd shapes such as $T$ shape normally not recommended for seismic safety point of view as the line of flange and web of $\mathrm{T}$ sec. may exhibit erratic kind of development of forces due to significant variation in the flange \& web.

2) Part of $T$ sec. plan bringing rotational flexibility brings special advantage in reducing the forces in column away from there entered corner columns, however there can be higher moments in outer columns. While introducing rotational flexibility a great care is desired in critical analysis of displacement and forces in flange section.

References

[1]. Mohit Sharma Dr. Savita Mar Dynamic analysis of multistoried regular building” (Jan. 2014)

[2]. Rakesh Sakale, R K Arora1 and Jitendra Chouhan "Seismic behavior of the building having horizontal irregularities" November 2014

[3]. Rucha S. Banginwar, M. R. Vyawahare " Effect of plans configurations on the seismic behavior of the structure by response spectrum method "May-Jun 2012

[4]. Divyashree M, Gopi Siddappa "Seismic behavior of RC buildings with re-entrant corners and strengthening "

[5]. Karaesmen E., Types of structural damages and factors that increase additional damages (in Turkish), association of Turkish contractor's publications 1996:25-6.

[6]. Seymen A., Akın D., "Disaster management system of Turkey and lessons learned from 1999 big earthquakes", Ministry of public works and settlement general directorate of disaster affairs,

[7]. R. Kumar, Y. Singh, R. Deoliya "Effect of design and detailing deficiencies on seismic performance and vulnerability of Indian RC frame building" 